\title{
Riboswitches: choosing the best platform
}

Kristine B. Arnvig (k.arnvig@ucl.ac.uk)

Institute for Structural and Molecular Biology, University College London WC1E 6BT, UK

\begin{abstract}
Riboswitch discovery and characterisation has come a long way since the term was first coined almost two decades ago. Riboswitches themselves are likely derived from ancient ligand-binding transcripts, which have evolved into sophisticated genetic control elements that are widespread in prokaryotes. Riboswitches are associated with a multitude of cellular processes including biosynthetic pathways, transport mechanisms and stress responses leading to an ever-increasing appreciation for an in-depth understanding of their triggers and functions in order to address physiological and regulatory questions. The majority of riboswitches exert their control via transcriptional or translational expression platforms depending on their genetic context. It remains, however, to be determined precisely why one platform is favoured over another. Is this a question of the layout of the gene expression machinery, ligand availability, the degree of control required, serendipity or various combinations of these? With this review, rather than providing answers, I am hoping to plant a seed for further scientific discussions about this puzzle.
\end{abstract}

\section{Introduction}

Control of gene expression plays a fundamental role in the ability of all living organisms to adapt to changing environments. Naturally, this also applies to a pathogen's ability to adapt to ever-changing (host) environments, assaults from the immune system and possible drug treatment. Bacterial gene expression control involves a multi-layered and complex system of molecular interactions, starting with protein-protein and protein-DNA interactions during transcription initiation, followed by co- and post-transcriptional events, in which regulatory RNA plays a significant role e.g. $[1,2]$. This can be in the form of trans-acting antisense RNA and small regulatory RNAs (sRNAs), or cis-regulatory elements such as $5^{\prime}$ leaders and RNA switches. The latter employ a variety of ligands, structures and molecular mechanisms to control downstream gene expression in a wide range of Gram-positive (e.g. 
Bacillus subtilis) and Gram-negative (e.g. Escherichia coli) and other bacterial species, reviewed in e.g. [3-6]). Although the Gram classification is sometimes regarded as outdated, it does provide a quick reference to the type of cell envelope that surrounds a particular bacterial species as well as some of their cytoplasmic contents. However, there are several species that do not fit either category including the major human pathogen $M$. tuberculosis, for which experimental validation of predicted RNA switches is limited [7-9].

The majority of riboswitches known to date integrate with the main gene expression machineries of transcription and translation to exert their control, but it remains unclear how specific aptamers have become associated with either one or the other in different genetic contexts. This review puts forward some of the possible drivers of this differentiation, including mechanisms associated with termination of transcription in E. coli, B. subtilis and $M$. tuberculosis.

\section{Termination of transcription}

Termination of transcription in bacteria can largely be divided into factor independent (intrinsic) or factor dependent (mediated by Rho). Termination at the 3 ' end of genes or operons is an integral part of the transcription cycle; however, premature termination of transcription in the $5^{\prime}$ untranslated region (UTR) or early in the open reading frame (ORF), will naturally supress the expression of downstream genes. Combined with the option of antitermination (readthrough), this offers a point of co- and post-transcriptional control that is widespread in bacteria and fundamental to many RNA switches [10].

\section{Intrinsic termination}

The current model for intrinsic termination involves a GC-rich stretch of RNA, capable of forming a stable stem-loop structure, followed by a uracil (U)-rich tail (Fig. 1) [11]. The role of the poly-U tail is to $a$ ) induce pausing of the elongation complex long enough for the GCrich hairpin to form and $b$ ) to provide a weak DNA-RNA A:U (-rich) hybrid that facilitates hairpin formation; the poly- $U$ tail is therefore critical for termination and putative intrinsic terminators can often be identified by their poly-T(U) stretches [11].

According to predictions, there are significantly more intrinsic terminators in $B$. subtilis than in E. coli (340 per Mbp versus 200 per Mbp), while only a small fraction of $M$. tuberculosis genes contains this feature (20 per Mbp) $[12,13]$. It has therefore been suggested that a stem- 
loop alone, i.e. without a poly-U tail might be sufficient for termination in $M$. tuberculosis [13], but current research suggests that the mycobacterial RNA polymerase (RNAP), like the E. coli and B. subtilis RNAPs, requires a poly-U tail for intrinsic termination to occur $[8,14]$. Given the functional importance of the poly- $U$ tail for pausing and for hairpin formation, this seems plausible. However, the mycobacterial RNAP is able to terminate with certain 'imperfect' poly-U tails that are not recognised by the E. coli RNAP (Fig. 1), possibly with the aid of NusG and possibly due an inherent slower elongation rate of mycobacterial RNAP compared to both E. coli and B. subtilis RNAPs [14-16].

\section{Rho-dependent termination}

Factor- or Rho-dependent termination requires the binding of termination factor Rho (likely already bound to RNAP) to the nascent transcript during transcription elongation. Once bound, the RNA is threaded through the central pore of the Rho hexamer, until Rho makes contact with the RNA exit channel of the RNAP, after which the elongation complex is dissociated [11]. The Rho-binding sites on RNA (rut sites) span 80-90 nucleotides while the actual termination site is located between 10 and 100 nucleotides further downstream. The rut sites show little sequence conservation, but tend to be rich in cytosines, while low in guanines $[11,17]$. The coupling of transcription and translation in bacteria plays a crucial role in regulating/suppressing Rho-dependent termination and transcriptional polarity. As long as rut sites are masked by ribosomes, Rho is unable to gain access; however, if translation is interrupted and rut sites unmasked, Rho can bind and terminate transcription $[11,17]$. The termination of transcription as a consequence of the disruption of translation is referred to as (transcriptional) polarity.

While the function of Rho appears to be relatively well-conserved across bacterial species, the requirement for Rho is not. More specifically, rho is essential in E. coli and in $M$. tuberculosis $[18,19]$. In $B$. subtilis, Rho is expressed at very low levels and the gene is not essential, but in fact entirely absent in some Firmicutes $[19,20]$. Together with the predicted frequency of intrinsic terminators in the three species, this suggests that $E$. coli and $M$. tuberculosis share the trait of relying more on Rho-dependent termination than B. subtilis, which relies more on intrinsic termination. 


\section{Additional factors modulating termination}

Factors intimately involved in both types of termination include NusA and NusG. NusA is essential in all three species (in wildtype background) [21-23], and is critical for transcriptional pausing and RNA folding in addition to promoting intrinsic termination $[11,24$, 25]. The nusG gene is essential in $M$. tuberculosis according to transposon mutagenesis [22]. In $E$. coli however, nusG is only essential in strains containing foreign DNA such as the rac prophage, where it is believed to assist Rho-mediated silencing of toxic genes $[26,27]$. nusG is not essential in B. subtilis, which may be linked to the fact that Rho is also not essential in B. subtilis $[21,22,27]$. Curiously, while NusG stimulates Rho-dependent termination in E. coli it stimulates intrinsic termination in M. tuberculosis [11].

\section{Riboswitches}

The ability of RNA to form different and alternative base-pairs within the same molecule, enables the formation of complex three-dimensional structures that can change depending on the immediate environment of the transcript. RNA switches exploit this by adopting dramatically different (permissive or non-permissive) conformations depending on the presence (concentration) of highly specific ligands ranging in size from metal ions to tRNAs $[5,6]$. The term 'riboswitch' is mostly associated with RNA switches that recognise smaller molecules without the involvement of either protein or additional RNA molecules such as tRNAs [4]. Riboswitches, typically located in the 5' UTR of the mRNA they regulate, are comprised of a ligand binding aptamer domain connected via a switching sequence to an expression platform, implementing gene expression control [5, 28-30]. The overall structure of aptamer domains is conserved through covariance, while the individual nucleotides that interact directly with specific ligands, are more highly conserved [5, 31]. Binding of a cognate ligand can lead to increased expression (ON-switches) or decreased expression (OFFswitches). It is however likely that regulation is rarely (if ever) a question of completely ON or OFF; rather these elements provide a fine-tuning that is critical to maintaining optimal concentrations of individual metabolites without large fluctuations [32, 33].

The most abundant types of expression platforms are the transcriptional (regulating via termination/antitermination) and the translational (regulating by masking/unmasking of ribosome binding sites (RBS) [4]. Due to the above-mentioned polarity, translational expression platforms may also involve Rho-dependent termination of transcription. 
Moreover, a few riboswitches have been shown to contain rut sites that can be masked/unmasked, meaning that Rho plays a prominent role in the control implemented by many riboswitches $[34,35]$. In effect this means, that the vast majority of known riboswitches regulate via either intrinsic or Rho-dependent termination of transcription.

\section{Exchanging one expression platform for another}

An intriguing feature of riboswitches is that individual, highly conserved aptamer domains can be associated with different types of expression platforms, usually transcriptional or translational, depending on gene and/or species context. Moreover, certain species prefer one over the other [31,33]. For example, riboswitches with conserved aptamer domains that recognise identical ligands tend to be associated with transcriptional expression platforms in B. subtilis and translational expression platforms in E. coli $[31,34]$. How this differentiation evolved is poorly understood, but comparative studies performed on the widespread FMN (flavin mononucleotide) riboswitch may provide some clues. In E. coli this switch controls expression of a single gene ( $r i b B)$ via a dual transcriptional/translational expression platform followed by Rho-dependent termination of transcription [34, 35]. In $B$. subtilis the same riboswitch controls the FMN biosynthetic operon (ribDGEABHT) via a transcriptional OFF-switch (ribD) and a single FMN transport gene via a translational OFFswitch (ribU; the role of Rho is unknown in this case) [36]. Apart from the anti-RBS, the regions involved in the switching are highly conserved between the two elements (Fig. 2).

Does this suggest that an ancestral FMN riboswitch employed dual transcriptionaltranslational regulation with subsequent loss of either one or the other type of platform over time? If one assumes that some riboswitches date back to an ancient RNA world [37] and therefore predate template-encoded protein synthesis, i.e. translation, then transcriptional expression platforms should predate translational platforms at least for a number of 'older' switches. Further investigations on conserved aptamers with variable expression platforms in a range of species may shed further light on this conundrum, keeping in mind that on a systems level additional factors such as e.g. the RibR protein counteracting regulation by FMN, or riboswitches acting in trans as sRNAs may also affect global regulatory outcomes [38, 39]. 
Questions remain: when is a transcriptional platform more suitable than a translational platform, and what were the drivers that resulted in a consistent species-specific use of one platform over the other? The answer is likely the result of multiple parameters including life style, genetic context, growth conditions, intracellular and extracellular environment (ligand availability) and general configuration of gene expression machineries (i.e. transcriptional and translational machineries and dominant terminator type).

Notable and critical differences between the two platforms are that while a translationally controlled fold is reversible (at least until transcription is terminated by Rho) a transcriptionally regulated riboswitch requires a ligand-dependent 'conformational decision' before the formation of the terminator/antiterminator structure, i.e. co-transcriptionally. This in turn requires appropriate NusA-dependent pausing of the elongation complex, combined with ligand concentrations that are higher than the $K_{D}$ for the aptamer and higher than what is required for translationally controlled riboswitch to ensure an appropriate response $[24,40,41]$. There may also be some element of 'cost-benefit' associated with the different platforms. As already mentioned, Rho is scarce in B. subtilis and translational control combined with inefficient Rho-dependent termination of transcription might involve costly synthesis and degradation of mRNAs that are not translated. This would be particularly pertinent in large operons (e.g. ribDGEABHT), while perhaps less so in mono-cistronic operons (e.g. ribU). However, this still does not explain why an organism like E. coli, which otherwise makes ample use of intrinsic termination, favours translational platforms.

Very few M. tuberculosis riboswitches have been experimentally validated, but scrutiny of regions associated with conserved riboswitch aptamers reveals a lack of canonical intrinsic terminators, suggesting that $M$. tuberculosis tends to favour translational expression platforms similar to $E$. coli, which is perhaps not surprising, given the overall paucity of intrinsic terminators in $M$. tuberculosis.

Is expression platform linked to envelope permeability?

At this point it is worth recalling what traditionally classifies a bacterium as Grampositive or Gram-negative. This division of bacteria is largely based on their distinct ability to retain crystal violet (or not) during staining, an ability that is closely linked to the cell envelope, i.e. cell membrane(s) and cell wall, which differs significantly between the two groups. 
The Gram-positive envelope consists of a cytoplasmic membrane and a cell wall, while the Gram-negative cell envelope has an additional outer membrane. Hence, the former is generally considered more permeable than the latter, for which first point of entry for many nutrients and metabolites is via outer membrane porins or TonB dependent transporters [4244].

Similar to E. coli, the M. tuberculosis cell envelope comprises a highly impermeable twomembrane system with peptidoglycan as well as arabinogalactan between membranes. The outer membrane is anchored to the arabinogalactan via mycolic acids [45, 46]. Thus, in addition to sharing a requirement for Rho and a preference for translational expression platforms, E. coli and M. tuberculosis share the characteristic of a cell envelope that is much less permeable than that of $B$. subtilis.

Based on the following assumptions, it is perhaps worth considering if envelope permeability, ligand availability and response time have influenced the choice of expression platform (Fig. 3):

- A switch with a given aptamer domain requires higher concentration of ligand to promote switching of a transcriptional expression platform than a translational expression platform [41].

- High intracellular concentrations are reached faster by rapid assimilation of ligand.

- The Gram-positive cell envelope is generally more permeable than the Gram-negative allowing faster assimilation of some extracellular molecules.

There are always exceptions, one being Fusobacterium nucleatum, which has a Gramnegative cell envelope, but to a large extent a Gram-positive interior. This bacterium appears to favour the transcriptional platform, although sample size as well as the unusual makeup of this species makes this generalisation somewhat uncertain $[31,47]$. Would we perhaps learn more about riboswitch mechanisms by studying non-canonical expression platforms in the different species, i.e. transcriptionally controlled switches in E. coli and M. tuberculosis and translationally controlled switches in B. subtilis? 


\section{Different riboswitches regulating the expression of homologous proteins}

One step further in the shuffling of expression platforms can be observed in two riboswitches from $M$. tuberculosis. While regulating the expression of homologous genes, their mode of action is very different in many aspects. The first is the widely conserved ydaO switch regulating the expression of resuscitation promoting factor (Rpf) A. The second is the much less widespread $r p f B$ switch regulating the expression of the $r p f B-k s g A-i s p E$ operon, encoding a second Rpf (B), in addition to an RNA methyltransferase and an essential kinase involved in cell wall synthesis (Fig. 4) $[8,48]$. The expression pattern of the $r p f A$ and $r p f B$ genes is similar over a range of growth conditions (Table 1) [49], but the riboswitches regulating $r p f A$ and $r p f B$ are vastly different.

Judging by the absence of a canonical intrinsic terminator, ydaO employs the translational platform generally preferred by M. tuberculosis, while rpfB has been shown experimentally to employ a transcriptional platform $[8,50]$. Although not yet experimentally validated, the $M$. tuberculosis ydaO switch is predicted to be a cyclic-di-AMP sensing OFF switch, similar to the ones found in B. subtilis and Streptomyces coelicolor [50-52]. Whether this leads to Rho-dependent termination of transcription remains to be seen. As a cognate ligand has not yet been identified for the rpfB switch, it has not been possible to determine for certain if this is an ON or OFF switch, but based on predicted folds and free energy, it is assumed to be an ON switch [8].

Since there is no apparent conservation between the $r p f A$ and $r p f B 5^{\prime}$ UTRs, and since the elements show very different conservation patterns, i.e. widespread versus 'rare' (ancient versus more recent?), the two switches are likely to have evolved independently and possibly function in a complementary fashion. An interesting characteristic of the two platforms is the partial similarity to the $B$. subtilis FMN switch. In both cases, the translational platform is associated with a monocistronic operon $(r p f A)$, while the transcriptional platform is associated with a polycistronic operon ( $r p f B-k s g A-i s p E)$. Is this coincidental or does it provide a clue to another driver of platform divergence?

\section{Final remarks}

Our knowledge of RNA- and riboswitch abundance, structure, mechanisms and importance has come a long way from their initial identification almost two decades ago. This is documented by more than 1300 reports that have provided comprehensive insights into 
how riboswitches are capable of sensing and converging a multitude of environmental inputs into a regulatory outcome. This review reflects on some, but far from all of the parameters that over time may have contributed to the extant make-up of different riboswitches in different organisms. Some suggestions are admittedly more speculative than others and overall these do not cover all the possible inputs, but rather serve to illustrate that we still have much more to learn about these elements. The dramatic increase in riboswitch knowledge has in many cases been driven by computational predictions employing sequence and structural comparisons in an extensive range of species. However, recently developed approaches based on unbiased, experimental identification of conditional termination of transcription, e.g. Term-seq [53], holds promise of a new era for riboswitch discovery including the unusual, the unexpected and the rare.

\section{Perspectives}

- Riboswitches are cis-regulatory RNA element often involved in the regulation of essential genes making them potential antimicrobial drug targets.

- Riboswitches serve as useful additions to synthetic biology toolkits.

- Most known riboswitches comprise highly conserved aptamer domains associated with transcriptional or translational expression platforms.

- The species-specific bias towards one or the other expression platform is likely driven by multiple factors, one of which may be permeability of the cell envelope.

- Expanding riboswitch discovery to include less conserved elements and non-model organisms will further our understanding of the evolution, function and overall impact of these elements. 


\section{Acknowledgements}

I am grateful to Brian Ho, Ramesh Wigneshweraraj and Finn Werner for critical reading of the manuscript.

\section{Funding}

KBA is funded by the Medical research Council MR/S009647/1

\section{References}

1. Beisel, C.L. and G. Storz, Base pairing small RNAs and their roles in global regulatory networks. FEMS Microbiol Rev, 2010. 34(5): p. 866-82.

2. Nitzan, M., R. Rehani, and H. Margalit, Integration of Bacterial Small RNAs in Regulatory Networks. Annu Rev Biophys, 2017. 46: p. 131-148.

3. Bastet, L., et al., Maestro of regulation: Riboswitches orchestrate gene expression at the levels of translation, transcription and mRNA decay. RNA Biol, 2018. 15(6): p. 679682.

4. Breaker, R.R., Riboswitches and Translation Control. Cold Spring Harb Perspect Biol, 2018. 10(11).

5. McCown, P.J., et al., Riboswitch diversity and distribution. RNA, 2017. 23(7): p. 9951011.

6. Sherwood, A.V. and T.M. Henkin, Riboswitch-Mediated Gene Regulation: Novel RNA Architectures Dictate Gene Expression Responses. Annu Rev Microbiol, 2016. 70: p. 361-74.

7. Arnvig, K.B., et al., Sequence-based analysis uncovers an abundance of non-coding RNA in the total transcriptome of Mycobacterium tuberculosis. PLoS Pathog, 2011. 7(11): p. e1002342.

8. Schwenk, S., et al., Cell-wall synthesis and ribosome maturation are co-regulated by an RNA switch in Mycobacterium tuberculosis. Nucleic Acids Res, 2018. 46(11): p. 5837-5849.

9. Warner, D.F., et al., A riboswitch regulates expression of the coenzyme B12independent methionine synthase in Mycobacterium tuberculosis: implications for differential methionine synthase function in strains H37Rv and CDC1551. J Bacteriol, 2007. 189(9): p. 3655-9.

10. Millman, A., et al., Computational prediction of regulatory, premature transcription termination in bacteria. Nucleic Acids Res, 2017. 45(2): p. 886-893.

11. Ray-Soni, A., M.J. Bellecourt, and R. Landick, Mechanisms of Bacterial Transcription Termination: All Good Things Must End. Annu Rev Biochem, 2016. 85: p. 319-47.

12. de Hoon, M.J., et al., Prediction of transcriptional terminators in Bacillus subtilis and related species. PLoS Comput Biol, 2005. 1(3): p. e25.

13. Unniraman, S., R. Prakash, and V. Nagaraja, Alternate paradigm for intrinsic transcription termination in eubacteria. J Biol Chem, 2001. 276(45): p. 41850-5.

14. Czyz, A., et al., Mycobacterial RNA polymerase requires a U-tract at intrinsic terminators and is aided by NusG at suboptimal terminators. MBio, 2014. 5(2): p. e00931. 
15. Chamberlin, M.J., et al., A quantitative assay for bacterial RNA polymerases. J Biol Chem, 1979. 254(20): p. 10061-9.

16. Harshey, R.M. and T. Ramakrishnan, Rate of ribonucleic acid chain growth in Mycobacterium tuberculosis H37Rv. J Bacteriol, 1977. 129(2): p. 616-22.

17. Boudvillain, M., N. Figueroa-Bossi, and L. Bossi, Terminator still moving forward: expanding roles for Rho factor. Curr Opin Microbiol, 2013. 16(2): p. 118-24.

18. Botella, L., et al., Depleting Mycobacterium tuberculosis of the transcription termination factor Rho causes pervasive transcription and rapid death. Nat Commun, 2017. 8: p. 14731.

19. D'Heygere, F., M. Rabhi, and M. Boudvillain, Phyletic distribution and conservation of the bacterial transcription termination factor Rho. Microbiology, 2013. 159(Pt 7): p. 1423-36.

20. Muntel, J., et al., Comprehensive absolute quantification of the cytosolic proteome of Bacillus subtilis by data independent, parallel fragmentation in liquid chromatography/mass spectrometry (LC/MS(E)). Mol Cell Proteomics, 2014. 13(4): p. 1008-19.

21. Ingham, C.J., J. Dennis, and P.A. Furneaux, Autogenous regulation of transcription termination factor Rho and the requirement for Nus factors in Bacillus subtilis. Mol Microbiol, 1999. 31(2): p. 651-63.

22. Sassetti, C.M., D.H. Boyd, and E.J. Rubin, Genes required for mycobacterial growth defined by high density mutagenesis. Mol Microbiol, 2003. 48(1): p. 77-84.

23. Zheng, C. and D.I. Friedman, Reduced Rho-dependent transcription termination permits NusA-independent growth of Escherichia coli. Proc Natl Acad Sci U S A, 1994. 91(16): p. 7543-7.

24. Lemay, J.F., et al., Comparative study between transcriptionally-and translationallyacting adenine riboswitches reveals key differences in riboswitch regulatory mechanisms. PLoS Genet, 2011. 7(1): p. e1001278.

25. Pan, T., et al., Folding of a large ribozyme during transcription and the effect of the elongation factor NusA. Proc Natl Acad Sci U S A, 1999. 96(17): p. 9545-50.

26. Cardinale, C.J., et al., Termination factor Rho and its cofactors NusA and NusG silence foreign DNA in E. coli. Science, 2008. 320(5878): p. 935-8.

27. Downing, W.L., et al., Sequence and transcriptional pattern of the essential Escherichia coli secE-nusG operon. J Bacteriol, 1990. 172(3): p. 1621-7.

28. Barsacchi, M., et al., SwiSpot: modeling riboswitches by spotting out switching sequences. Bioinformatics, 2016. 32(21): p. 3252-3259.

29. Breaker, R.R., Prospects for riboswitch discovery and analysis. Mol Cell, 2011. 43(6): p. 867-79.

30. Nudler, E. and A.S. Mironov, The riboswitch control of bacterial metabolism. Trends Biochem Sci, 2004. 29(1): p. 11-7.

31. Barrick, J.E. and R.R. Breaker, The distributions, mechanisms, and structures of metabolite-binding riboswitches. Genome Biol, 2007. 8(11): p. R239.

32. Bennett, B.D., et al., Absolute metabolite concentrations and implied enzyme active site occupancy in Escherichia coli. Nat Chem Biol, 2009. 5(8): p. 593-9.

33. Ray, S., A. Chauvier, and N.G. Walter, Kinetics coming into focus: single-molecule microscopy of riboswitch dynamics. RNA Biol, 2018: p. 1-9. 
34. Bastet, L., et al., Translational control and Rho-dependent transcription termination are intimately linked in riboswitch regulation. Nucleic Acids Res, 2017. 45(12): p. 74747486.

35. Hollands, K., et al., Riboswitch control of Rho-dependent transcription termination. Proc Natl Acad Sci U S A, 2012. 109(14): p. 5376-81.

36. Winkler, W.C., S. Cohen-Chalamish, and R.R. Breaker, An mRNA structure that controls gene expression by binding FMN. Proc Natl Acad Sci U S A, 2002. 99(25): p. 15908-13.

37. Breaker, R.R., Riboswitches and the RNA world. Cold Spring Harbor perspectives in biology, 2012. 4(2).

38. Loh, E., et al., A trans-acting riboswitch controls expression of the virulence regulator PrfA in Listeria monocytogenes. Cell, 2009. 139(4): p. 770-9.

39. Pedrolli, D.B., et al., A dual control mechanism synchronizes riboflavin and sulphur metabolism in Bacillus subtilis. Proc Natl Acad Sci U S A, 2015. 112(45): p. 14054-9.

40. Steinert, $\mathrm{H}$., et al., Pausing guides RNA folding to populate transiently stable RNA structures for riboswitch-based transcription regulation. Elife, 2017. 6.

41. Wickiser, J.K., et al., The speed of RNA transcription and metabolite binding kinetics operate an FMN riboswitch. Mol Cell, 2005. 18(1): p. 49-60.

42. Dam, S., J.M. Pages, and M. Masi, Stress responses, outer membrane permeability control and antimicrobial resistance in Enterobacteriaceae. Microbiology, 2018. 164(3): p. 260-267.

43. Schauer, K., D.A. Rodionov, and H. de Reuse, New substrates for TonB-dependent transport: do we only see the 'tip of the iceberg'? Trends Biochem Sci, 2008. 33(7): p. 330-8.

44. Silhavy, T.J., D. Kahne, and S. Walker, The bacterial cell envelope. Cold Spring Harb Perspect Biol, 2010. 2(5): p. a000414.

45. Bansal-Mutalik, R. and H. Nikaido, Mycobacterial outer membrane is a lipid bilayer and the inner membrane is unusually rich in diacyl phosphatidylinositol dimannosides. Proc Natl Acad Sci U S A, 2014. 111(13): p. 4958-63.

46. Jackson, M., The mycobacterial cell envelope-lipids. Cold Spring Harb Perspect Med, 2014. 4(10).

47. Mira, A., et al., Evolutionary relationships of Fusobacterium nucleatum based on phylogenetic analysis and comparative genomics. BMC Evol Biol, 2004. 4: p. 50.

48. Block, K.F., M.C. Hammond, and R.R. Breaker, Evidence for widespread gene control function by the ydaO riboswitch candidate. J Bacteriol, 2010. 192(15): p. 3983-9.

49. Gupta, R.K., B.S. Srivastava, and R. Srivastava, Comparative expression analysis of rpflike genes of Mycobacterium tuberculosis H37Rv under different physiological stress and growth conditions. Microbiology, 2010. 156(Pt 9): p. 2714-22.

50. Schwenk, S. and K.B. Arnvig, Regulatory RNA in Mycobacterium tuberculosis, back to basics. Pathog Dis, 2018. 76(4).

51. Nelson, J.W., et al., Riboswitches in eubacteria sense the second messenger c-di-AMP. Nat Chem Biol, 2013. 9(12): p. 834-9.

52. St-Onge, R.J., et al., Nucleotide second messenger-mediated regulation of a muralytic enzyme in Streptomyces. Mol Microbiol, 2015. 96(4): p. 779-95.

53. Dar, D., et al., Term-seq reveals abundant ribo-regulation of antibiotics resistance in bacteria. Science, 2016. 352(6282): p. aad9822. 


\section{Figure legends}

Fig. 1: Schematic illustrating different types of intrinsic terminators mentioned in the text. A canonical terminator is characterised by a stable stem-loop structure immediately followed by a poly-U tail. An imperfect $U$-tail has one or more non- $U$ residues inserted in the tail. $A$ stem-loop without a poly-U tail may not be functional as a terminator.

Fig. 2: Alignment of ribU and ribD FMN switches from B. subtilis. Regions involved in transcriptional regulation are highlighted in green and red and translational regulation in yellow and orange. The alignment illustrates how the similarity between the two elements extends beyond the aptamer domain such that much of the sequence required for terminator formation is seen in the translational platform. Conversely, the entire region involved in sequestration of the ribosome binding site (Anti-RBS) is unique to the ribU switch.

Fig. 3: Is expression platform linked to envelope permeability? Schematic proposing how envelope permeability may affect assimilation of extracellular (or efflux of intracellular) ligands and riboswitch control. Beige broken lines indicate peptidoglycan, solid grey line, outer membrane. The upper cell represents a Gram-positive bacterium (e.g. B. subtilis), where extracellular metabolites are easily assimilated with a corresponding rapid increase in their intracellular concentrations; this scenario would facilitate transcriptional control, as transcriptional expression platforms require higher concentration of ligand to induce switching [41]. Bottom cell represents the less permeable Gram-negative and Acid-fast envelopes, where assimilation of ligand is restricted and its intracellular concentration likely increases more slowly compared to the Gram-positive cell. Hence, a translationally controlled switch may be more favourable to avoid irreversible decisions inconsistent with actual conditions.

Fig. 4: The $r p f B-k s g A-i s p E$ operon controlled by an RNA-switch in M. tuberculosis. The image shows the operon layout with its main transcription start site (black arrow) followed by an RNA switch/conditional terminator and the three coding regions.

Table 1: Comparison of selected characteristics of $r p f A$ and $r p f B$ 
